\title{
Radar Sounding of Glaciers in Greenland
}

\author{
C. Allen, B. Wohletz, and S. Gogineni \\ The University of Kansas, Radar Systems and Remote Sensing Laboratory \\ 2291 Irving Hill Drive \\ Lawrence, KS 66045 USA \\ TEL: (913) 864-3017, FAX: (913) 864-7789, E-mail: callen@eecs.ukans.edu
}

\begin{abstract}
We have developed a coherent radar depth sounder operating at $150 \mathrm{MHz}$. The system is designed to operate as an unfocused SAR and obtains the sensitivity required to sound glacial ice through signal processing even though peak transmit power is about $200 \mathrm{~W}$. We installed this system on a NASA P-3 aircraft and collected radar data on several flights over Greenland glaciers during the summer of 1995 . The radar data were collected in conjunction with laser elevation measurements performed by NASA Wallops and are tagged with Global Positioning System (GPS) information. We collected data over the Humboldt, Jacobshavn and Petermann glaciers. To the best of our knowledge the radar data over these glaciers are the first ever obtained [1]. We applied a homomorphic deconvolution technique to remove multiple echoes of surface and ice-bottom interface. We have been able to identify the grounding line from the radar data. In this paper we provide a brief system description and experimental results along with geophysical interpretation of radar data. We also discuss the theory and present results of the homomorphic deconvolution procedure.
\end{abstract}

\section{INTRODUCTION}

The Greenland and Antarctic ice sheets together contain about $90 \%$ of the world's fresh water. The Greenland ice sheet, near equilibrium with its environment now, reached its maximum size about 15,000 years ago and has since retreated to its present size. By monitoring the mass balance of the ice sheets we can gain a better understanding of the effects of global warming on the ice sheets. The mass balance is determined by the annual precipitation, ablation, drainage at the edges, and iceberg calving. The volume of ice discharged into the sea by outlet glaciers is an important part of the total mass balance equation. To assess the ice flux in an outlet glacier, its thickness profile is needed as well as its flow rate. (Other techniques including spaceborne radar imaging have been shown to provide information on glacier flow rates [2].) While both radio echo sounding and seismic techniques have been used previously to measure the thickness of ice sheets with good agreement, radio echo sounding achieves much better range resolution and allows continuous recording of ice thickness data while seismic methods do not.

While many parts of the Antarctic and Greenland ice sheets have been successfully depth sounded, there have not been many successful radio-echo-sounding measurements made on outlet glaciers. Radio echo sounding of outlet glaciers is difficult because of absorption due to warm ice, scattering from water, or impurities in the ice, and because of reflections from nearby valley sides.

During the summer of 1995 , we performed airborne radar echo soundings of several glaciers in Greenland. From our data we can determine glacier thickness, the location of the grounding line, and the ice thickness at the calving front. These soundings are the first successful radar sounding data collected from these glaciers [1].

\section{SYSTEM DESCRIPTION}

The radar depth sounder we used in this study is a modified and improved version of the Coherent Antarctic Radar Depth Sounder (CARDS) designed and build by the Radar Systems and Remote Sensing Laboratory (RSL) at The University of Kansas [3]. Modifications made to the original CARDS include an improved sensitivity time control (STC), a reduction in the noise level, improved isolation between the transmitter and the receiver, improved surface acoustic wave (SAW), and a new antenna structure resulting in less turbulence. This system was operated from a NASA P-3 aircraft that was also equipped with precision laser altimeter systems and Global Positioning System (GPS) receivers. Consequently, the radar data are tagged with precise GPS location information, providing accurate registration of sounding data with sensor position and an independent measurement of the ice surface elevation.

Our airborne, coherent radar system operates at a center frequency of $150 \mathrm{MHz}$. Operating at a pulse repetition frequency (PRF) of $9800 \mathrm{~Hz}$, the transmitter generates a pulse that is frequency modulated (chirped) over a bandwidth of $17 \mathrm{MHz}$ with a duration of $1.6 \mu \mathrm{s}$ and a peak power of $200 \mathrm{~W}$. The receiver, protected during transmit events by a blanking switch, compresses the received signal in a weighted SAW compressor resulting in a compressed pulse length of $60 \mathrm{~ns}$ and a depth resolution of $5 \mathrm{~m}$ in ice $(n=1.78)$. The received signal is next downconverted to baseband via coherent detection providing in-phase and quadrature ( $I$ and $Q$ ) analog outputs that are digitized in pairs with two 8-bit A/D converters at a rate of 18.75 megasamples/sec (MSPS). Coherent integration is then performed by summing complex data vectors from 256 consecutive transmit-receive periods. The data are then 
detected by computing the power in each record $\left(\mathrm{I}^{2}+\mathrm{Q}^{2}\right)$ and then integrated further (incoherent integration) by summing four consecutive detected signals. The data are finally displayed to the user and are also recorded (along with position and time data provided by the on-board GPS receiver) at a rate of about $9 \mathrm{kbytes} / \mathrm{sec}$ on a removable hard disk.

Separate transmit and receive antennas are mounted beneath the left and right wings, with each antenna being a four-element, half-dipole array. The theoretical two-way, half-power beamwidth of this antenna configuration is about $18^{\circ}$ in the plane normal to the flight path and about $66^{\circ}$ in the plane parallel to the flight path. The coherent integration serves as a low-pass filter on the data and is equivalent to reducing the along-track antenna beamwidth from about $66^{\circ}$ to $7^{\circ}$ at the nominal velocity.

We believe the pulse compression, coherent processing and the bistatic antenna arrangement are the features that permitted this system to succeed in sounding these glaciers.

\section{EXPERIMENT DESCRIPTION}

In May 1995, an overflight of the Petermann glacier was flown principally along its flow line. This flight line is continuous, extending from the Greenland ice sheet to the ocean. Fig. 1 shows the flight line flown over the Petermann glacier. During data acquisition, the aircraft altitude was approximately $500 \mathrm{~m}$ above local terrain and the air speed was about $130 \mathrm{~m} / \mathrm{s}$.

\section{DATA PROCESSING}

When the data were analyzed, in addition to observing echoes from the surface of the ice and from the ice-rock interface at the bottom of the glacier, we sometimes observed numerous replicas of these echoes from the strongly scattering surfaces, that the range between these multiple echoes was relatively constant, and that this range corresponded to the altitude of the aircraft above the ice surface. We concluded that these artifacts resulted from a multipath phenomenon involving reflections from the ice surface and the underside of the aircraft.

To remove these artifacts without removing other real features in the data, we applied homomorphic deconvolution, wherein a signal composed of the convolution of two signals can be converted to a signal space where the two signals are now summed. In our case, we have a record of data that can be represented by the convolution of our pulse (and its delayed image) with a series of impulses corresponding with the reflection characteristics of the ice and bedrock. Oppenheim and Schmidt [4] developed much of the theory behind homomorphic deconvolution. Essentially, by transforming our data first to the frequency domain (transforming convolutional processes into multiplicative

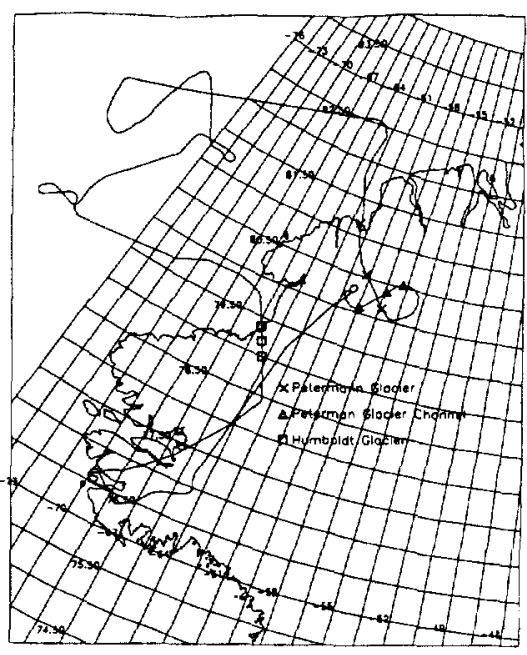

Fig. 1 Flight lines over the Petermann glacier.

ones) and then taking the complex logarithm of this frequency domain data (transforming multiplicative processes into additive ones), we are able to subtract the multiple reflection components without adversely affecting the remainder of the data. Returning to the original time domain of our data requires an undoing of the sequence just described (i.e., performing a complex exponential and then transformation from the frequency domain to the time domain via Fourier transform).

To separate successfully the multiple reflections from the desired data, the time-domain data must be either a minimalphase or a maximal-phase sequence; however our data were neither, it was a mixed-phase sequence. Therefore we converted it into a minimal-phase sequence by multiplying our data sequence by an exponential weighting prior to applying the deconvolution process [5]. Removal of this exponential weighting is then required following the completion of the deconvolution.

\section{RESULTS}

Fig. 2 (top) shows the radio echogram from the Petermann glacier prior to homomorphic deconvolution. In the left half of this echogram, the return from the surface and the bottom are clearly identifiable. Also seen is a multiple echo resulting from a multipath between the ice surface and the aircraft. The distance between the true return from the ice surface and its multiple echo is approximately 60 pixels, and, while each pixel corresponds to 4.49 meters in ice, in air each pixel corresponds to 8 meters so that the separation between the true and multiple echoes is about 480 meters, which corresponds to the nominal aircraft altitude of 500 meters. In the right half of this echogram we see numerous multiple echoes again arising from the ice surface-aircraft multipath, except here we are also seeing multiples of the bottom return as it is also a relatively strong echo at the shallower depths. 

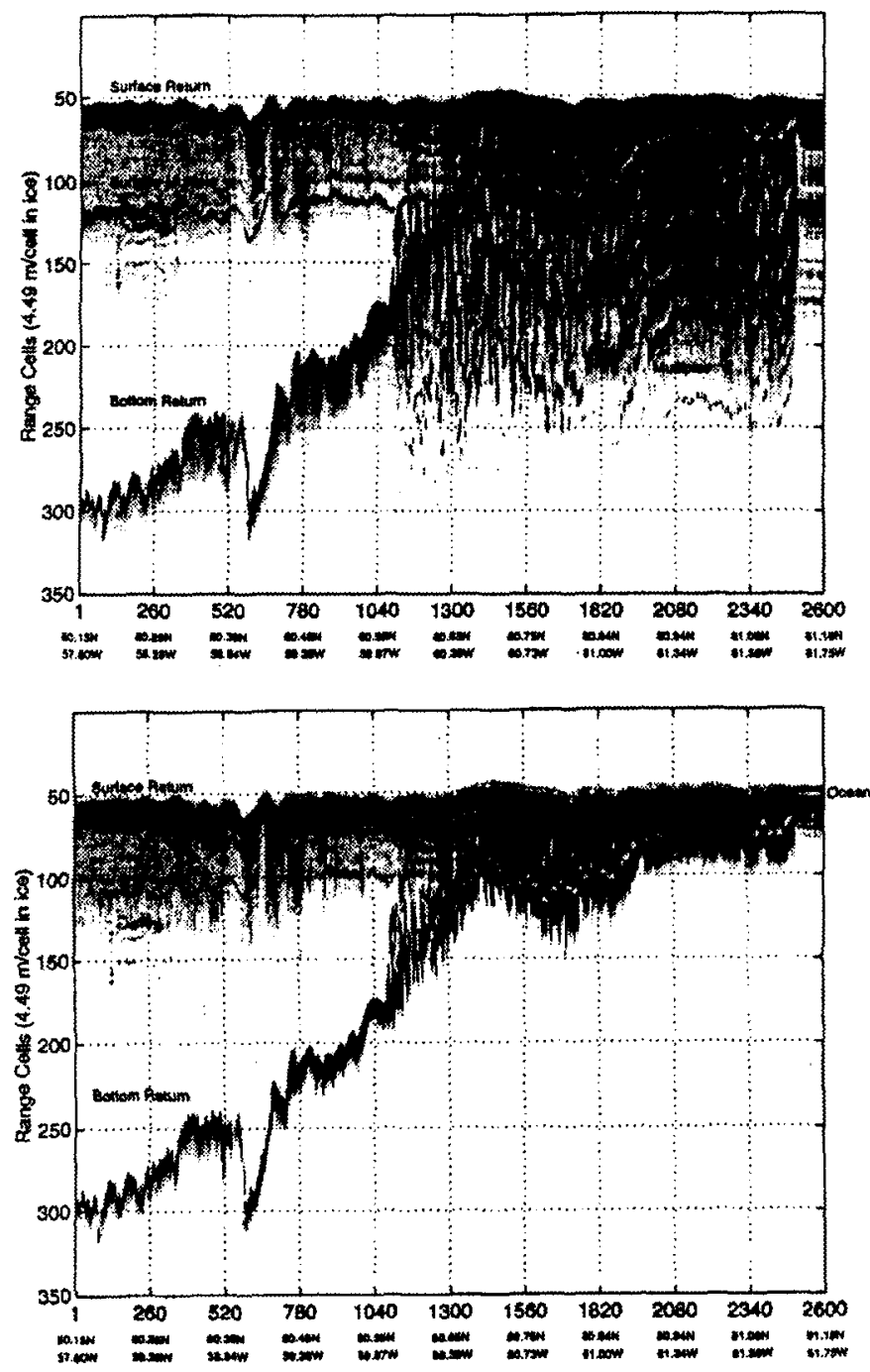

Fig. 2 Radio echogram of the Petermann glacier before (above) and after homomorphic deconvolution (below).

The distance between these multiple echoes is again approximately 60 pixels, supporting the ice-aircraft multipath hypothesis. Unambiguous determination of the ice thickness from these data is difficult at best.

In the lower part of Fig. 2 we see the effects of the homomorphic deconvolution. The multiple echo from the surface in the left half is removed and the numerous echoes in the right half are also removed, making the determination of the ice thickness possible by finding the distance between the surface return and the bottom return. The ice thickness of the Petermann glacier is presented in Fig. 3. In this plot we see a rapidly decreasing thickness as we approach the ocean, except for the valley at around $80.40 \mathrm{~N} / 58.60 \mathrm{~W}$. We believe the grounding line (where the glacier begins to float) may lie between points $80.59 \mathrm{~N} / 59.88 \mathrm{~W}$ and $80.65 \mathrm{~N} / 60.39 \mathrm{~W}$ as it appears that the glacier begins to experience bottom crevassing. The glacier thins rapidly immediately beyond the

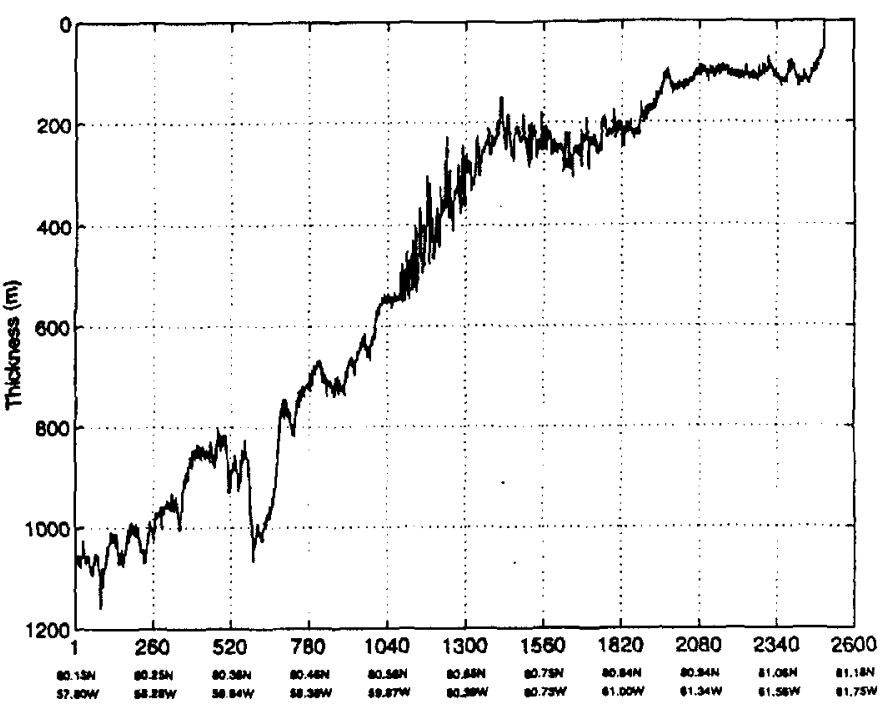

Fig. 3 Ice thickness of Petermann glacier.

grounding line but then it reaches a relatively constant thickness and at the calving front we see a thickness of $60 \mathrm{~m}$.

\section{CONCLUSIONS}

We have developed a coherent depth sounder that is capable of measuring the thickness of outlet glaciers. Multiple echoes arise from the ice surface-airplane multipath. By applying homomorphic deconvolution we were able to remove these multiple returns, enabling us to measure the thickness of the Petermann glacier from the Greenland ice sheet continuously to its calving front at the ocean. We have also detected evidence of the grounding line.

\section{REFERENCES}

[1] Weidick, A. "Jacobshavn Isbrae During the Climatic Optimum," Rapp. Gronlands Geolol. Unders., vol. 155, pp. 67-72, 1992.

[2] Joughlin, I., R. Kwok, M. Fahnestock, S. Gogineni, C. Allen, "Interferometrically Derived Topography, Velocity, and Ice-Flux Estimates for the Petermann Glacier," EOS, Transactions, American Geophysical Union, 1995 Fall Meeting, v. 76(46), p. F184, December 1995.

[3] Raju, G., W. Xin, and R. K. Moore, "Design, Development, Field Observations, and Preliminary Results of the Coherent Antarctic Radar Depth Sounder (CARDS) of the University of Kansas, U.S.A.," Journal of Glaciology, v. 36(123), pp. 247-254, 1990.

[4] Oppenheim, A. V. and R. W. Schafer, "Homomorphic Analysis of Speech," IEEE Transactions on Audio and Electroacousatics, AU-16(2), 1968.

[5] Schafer, R. W., "Echo Removal by Discrete Generalized Linear Filtering," Research Laboratory of Massachusetts Institute of Technology, Technical Report 466, 1969. 